\title{
ON REDUCED SCALAR EQUATIONS FOR SYNCHRONOUS BOOLEAN NETWORKS
}

\author{
Ali Muhammad Ali Rushdi and Adnan Ahmad Alsogati \\ Department of Electrical and Computer Engineering, Faculty of Engineering, \\ King Abdulaziz University, P.O. Box 80204, Jeddah 21589, Saudi Arabia
}

Received 2013-08-22, Revised 2013-09-09; Accepted 2013-09-10

\begin{abstract}
A total description of a synchronous Boolean network is typically achieved by a matrix recurrence relation. A simpler alternative is to use a scalar equation which is a possibly nonlinear equation that involves two or more instances of a single scalar variable and some Boolean operator(s). Further simplification is possible in terms of a linear reduced scalar equation which is the simplest two-term scalar equation that includes no Boolean operators and equates the value of a scalar variable at a latter instance $t_{2}$ to its value at an earlier instance $t_{1}$. This equation remains valid when the times $t_{1}$ and $t_{2}$ are both augmented by any integral multiple of the underlying time period. In other words, there are infinitely many versions of a reduced scalar equation, any of which is useful for deducing information about the cyclic behavior of the network. However, to obtain correct information about the transient behavior of the network, one must find the true reduced scalar equation for which instances $t_{1}$ and $t_{2}$ are minimal. This study investigates the nature, derivation and utilization of reduced scalar equations. It relies on Boolean-algebraic manipulations for the derivation of such equations and suggests that this derivation can be facilitated by seeking certain orthogonality relations among certain successive (albeit not necessarily consecutive) instances of the same scalar variable. We demonstrate, contrary to previously published assumptions or assertions, that there is typically no common reduced scalar equation for all the scalar variables. Each variable usually satisfies its own distinct reduced scalar equation. We also demonstrate that the derivation of a reduced scalar equation is achieved not only by proving it but also by disproving an immediately preceding version of it when such a version might exist. We also demonstrate that, despite the useful insight supplied by the reduced scalar equations, they do not provide a total solution like the one offered by matrix methods and therefore they need be supported by other techniques of mathematical reasoning. We present three classical examples to illustrate our techniques. Two examples are tutorials on the necessary Boolean-algebraic techniques. They present corrections of previously published results and refute purported claims of discrepancies between scalar and matrix methods. The third example illustrates how the reduced scalar equations can be supplemented by techniques of number theory, Diophantine equations and Boolean equations in making subtle inferences about Boolean networks. We achieved a better understanding of the nature of reduced scalar equations, demonstrated Boolean-algebraic techniques for deriving them, presented other mathematical tools for utilizing them and finally reconciled them with the more encompassing but more complex and less insightful matrix methods.
\end{abstract}

Keywords: Synchronous Boolean Networks, Distinct Reduced Scalar Boolean Equations, Orthogonality, Proof and Disproof, Diophantine Equations, Boolean Equations

\section{INTRODUCTION}

A Synchronous Boolean network is a set of $n$ nodes, each of which is either in state 1 (on) or state 0 (off) at any given time $t$. Each node is updated at time $(t+1)$ by inputs from any fixed subset of the set of nodes according to any desired logical rule. Since the total number of possible network states is finite $\left(2^{\mathrm{n}}\right)$ and the

Corresponding Author: Ali Muhammad Ali Rushdi, Department of Electrical and Computer Engineering, Faculty of Engineering, King Abdulaziz University, P.O. Box 80204, Jeddah 21589, Saudi Arabia 
network changes states sequentially in discrete time steps, the network must necessarily return to a previously occupied state in a finite time (at most $2^{\mathrm{n}}$ time points). This means that all possible trajectories of the network consist of either cycles (loops or attractors) of any length from size one (a fixed point) to a maximum of $2^{n}$, or transient states leading eventually to a cycle. An ideal total description of the network (in which one accounts for all $2^{\mathrm{n}}$ states) can be achieved by matrix methods (Brown, 2003; Cheng, 2009; Cheng and Qi, 2010a; 2010b; Cheng et al., 2011; Cull, 1971; Rushdi and AlOtaibi, 2007), but can be realized only for small $n$ and would be unfeasible for most networks of interest that usually have 100 or more nodes. Zhao (2005) showed that even the determination of the number of fixed points (cycles of length 1) for monotone Boolean networks and the determination of the existence of fixed points for general Boolean networks are both strong NP-complete problems, which means among other things that both problems are highly intractable and that the best algorithms that can ever be devised for them are highly inefficient.

Utilizing the earlier work of Darby and Mysak (1993) and Heidel et al. (2003) suggested that sometimes the matrix equations necessary to describe the logic of a given Boolean network can be reduced to a smaller set of higher-order scalar equations or even a single scalar equation and that such a scalar equation is more transparent to analyze for cycles, than the original matrix equations. The term "scalar equation" is used to denote an ordinary recurrence equation for a particular node of a Boolean network. This means that a scalar equation is an equation that involves two or more time instances of a single scalar variable and some Boolean operator(s), e.g., the equation $X_{1}(t+3)=1+X_{1}(t)$. As a sequel of Heidel et al. (2003) and Farrow et al. (2004) suggested that a linear reduced scalar equation be derived from the more rudimentary nonlinear scalar equation. The reduced scalar equation is a simpler but a higher-order equation. It is a two-term scalar equation that includes no Boolean operators and equates a latter instance of each scalar variable $\mathrm{X}_{\mathrm{i}}$ $(1 \leq \mathrm{i} \leq \mathrm{n})$ to an earlier instance of the same variable. The general form of the reduced scalar Equation (1) is:

$\mathrm{X}_{\mathrm{i}}\left(\mathrm{t}+\mathrm{r}_{\mathrm{i}}\right)=\mathrm{X}_{\mathrm{i}}\left(\mathrm{t}+\mathrm{s}_{\mathrm{i}}\right), 1 \leq \mathrm{i} \leq \mathrm{n}$

where $r_{i}$ and $s_{i}$ are the smallest integers such that $r_{i}>s_{i}$. Such a set of equations yields the immediate information that Equation (2 and 3):

Any possibal cycle length $=$ a divisor of $\left(\mathrm{r}_{\mathrm{i}}-\mathrm{s}_{\mathrm{i}}\right), 1 \leq \mathrm{i} \leq \mathrm{n}$
The longest possible transient trajectory $=\max _{\mathrm{i}}\left(\mathrm{s}_{\mathrm{i}}\right)$

Heidel et al. (2003) and Farrow et al. (2004) apparently assumed that $r_{i}$ and $s_{i}$ are the same for all $i$. This assumption is valid for most of the examples studied by them. However, it is not valid for the small 3variable example studied by Farrow et al. (2004) and Everest and Ward (2005), for which they asserted explicitly that all three nodes have exactly the same reduced scalar equation. The assumption of equality among all $\mathrm{r}_{\mathrm{i}} \mathrm{s}$ and all $\mathrm{s}_{\mathrm{i}} \mathrm{s}$ is invalid also for the major 11variable example studied by Farrow et al. (2004). In fact, the reduced scalar equation derived there in is neither minimal nor valid for all the network variables. That is why their value for the transient period of their example network does not agree with the corresponding value obtained by Cheng et al. (2011) via an exact matrix method based on a semi-tensor product approach.

This study is a continuation of the earlier work of Darby and Mysak (1993); Heidel et al. (2003) and Farrow et al. (2004). It strives to enhance the method of reduced scalar equations and reconcile its results with the more powerful (but less insightful) matrix methods.

\section{MATERIALS AND METHODS}

The methodology adopted herein is one of mathematical analysis and demonstration by way of examples. We utilize various techniques of switching algebra (two-valued Boolean algebra). We adhere to the linear representation (Reed-Müller expansion) of Boolean functions (Rushdi and Al-Otaibi, 2007). Therefore, we need to utilize well known properties of the Exclusive-OR (XOR) binary operator (+), also known as modulo-2 addition, or addition over the simplest finite or Galois Field GF(2) (Rushdi and Al-Otaibi, 2007). These properties include well known identities (Muroga, 1979; Gregg, 1998) such as Equation (4 to 8):

$X+X=0$

$\mathrm{X}+1=\overline{\mathrm{X}}$

$X+0=X$

$X+\bar{X}=1$

$X(1+X)=0$ 
We base our analysis on updating or iterating given equations and simplify our manipulations by seeking and utilizing certain orthogonality relations that exist among successive (albeit not necessarily consecutive) instances of the same scalar variable. We augment the reducedscalar-equation method by other methods of mathematical reasoning, including number theory, Diophantine equations and Boolean equations.

\section{RESULTS}

We demonstrate, contrary to previously published assumptions or assertions, that there is typically no common reduced scalar equation for all the scalar variables, i.e., $r_{i}$ and $s_{i}$ in (1) are not necessarily the same for all i. Each variable usually satisfies its own distinct reduced scalar equation. We also demonstrate that the derivation of a reduced scalar equation is achieved not only by proving it but also by disproving an immediately preceding version of it, when such a version could exist. In fact, one must prove (1) for the smallest integers $r_{i}$ and $s_{i}$, namely $r_{i m}$ and $s_{i m}$, where the extra subscript $m$ denotes "minimal". However, (1) is valid not only for $r_{i}=$ $\mathrm{r}_{\mathrm{im}}$ and $\mathrm{s}_{\mathrm{i}}=\mathrm{s}_{\mathrm{im}}$, but also for $\mathrm{r}_{\mathrm{i}}=\mathrm{r}_{\mathrm{im}}+\mathrm{k}$ and $\mathrm{s}_{\mathrm{i}}=\mathrm{s}_{\mathrm{im}}+\mathrm{k}$ where $\mathrm{k}$ is an arbitrary nonnegative integer. If $s_{i}=0$, then it is minimal and equals $s_{\mathrm{im}}$. Otherwise, a plausible way to guarantee that the smallest values $r_{i m}$ and $s_{i m}$ of $r_{i}$ and $\mathrm{S}_{\mathrm{i}}$ were attained is to prove Equation (9):

$\mathrm{X}_{\mathrm{i}}\left(\mathrm{t}+\mathrm{r}_{\mathrm{im}}\right)=\mathrm{X}_{\mathrm{i}}\left(\mathrm{t}+\mathrm{s}_{\mathrm{im}}\right), 1 \leq \mathrm{i} \leq \mathrm{n}$

and Equation (10):

$\mathrm{X}_{\mathrm{i}}\left(\mathrm{t}+\mathrm{r}_{\mathrm{im}}-1\right) \neq \mathrm{X}_{\mathrm{i}}\left(\mathrm{t}+\mathrm{s}_{\mathrm{im}}-1\right) \cdot 1 \leq \mathrm{i} \leq \mathrm{n}$

With the proof in (9) and the disproof in (10), one makes sure that (10) is indeed the true minimal reduced scalar equation, simply referred to as the reduced scalar equation.

Despite the useful insight supplied by the reduced scalar equations, they do not provide a total solution like the one offered by the more-powerful matrix methods and therefore they need be supported by other techniques of mathematical reasoning. The following examples illustrate the Boolean-algebraic techniques; necessary for the derivation of the reduced scalar equations, present corrections of previously published results, refute purported claims of discrepancies between scalar and matrix methods and illustrate how the reduced scalar equation can be supplemented by techniques of number theory, Diophantine equations and Boolean equations in making subtle inferences about Boolean networks.

\subsection{Example 1}

Figure 1 presents a 3-variables Boolean network studied by Farrow et al. (2004). For simplicity, the 3 nodes of the network are represented by the symbols A through C. Here, a solid arrow indicates a "positive" effect and a dotted arrow indicates a "negative" one. The network equations are as follows Equation (11a to c):

$$
\begin{aligned}
& \mathrm{A}(\mathrm{t}+1)=\mathrm{B}(\mathrm{t}) \mathrm{C}(\mathrm{t}) \\
& \mathrm{B}(\mathrm{t}+1)=1+\mathrm{A}(\mathrm{t}) \\
& \mathrm{C}(\mathrm{t}+1)=\mathrm{B}(\mathrm{t})
\end{aligned}
$$

Figure 2 shows a Karnaugh-map representation for the three next-state functions of $\mathrm{A}(\mathrm{t}+1), \mathrm{B}(\mathrm{t}+1)$ and $\mathrm{C}(\mathrm{t}+1)$ as functions of $\mathrm{A}(\mathrm{t}), \mathrm{B}(\mathrm{t})$ and $\mathrm{C}(\mathrm{t})$. For convenience, the current value of each map cell is shown in the top right corner of the cell. From Fig. 2, we obtain the state diagram in Fig. 3, which is a map of all possible trajectories among the $2^{3}=8$ states of the network. This diagram shows that the network has a single cycle of length five and two transient trajectories, the longest of which is of length two.

Since equations resulting from any increment in $t$ are also valid [An equation valid for $\mathrm{t}$ ia also valid for $\mathrm{t}+1, \mathrm{t}$ $+2, t+3, \ldots]$, we obtain Equation (12):

$\mathrm{A}(\mathrm{t}+2)=\mathrm{B}(\mathrm{t}+1) \mathrm{C}(\mathrm{t}+1)=(1+\mathrm{A}(\mathrm{t})) \mathrm{B}(\mathrm{t})$

Multiplying both sides of (12) by $\mathrm{A}(\mathrm{t})$, one obtains the orthogonality condition Equation (13):

$\mathrm{A}(\mathrm{t}) \mathrm{A}(\mathrm{t}+2)=0$

A further increment of t produces Equation (14):

$$
\begin{aligned}
\mathrm{A}(\mathrm{t}+3) & =(1+\mathrm{A}(\mathrm{t}+1)) \mathrm{B}(\mathrm{t}+1) \\
& =(1+\mathrm{A}(\mathrm{t}+1))(1+\mathrm{A}(\mathrm{t}))
\end{aligned}
$$

which is a scalar equation for $\mathrm{A}$ that implies the two orthogonality conditions Equation (15 and 16):

$$
\begin{aligned}
& A(t) A(t+3)=0 \\
& A(t+1) A(t+3)=0
\end{aligned}
$$




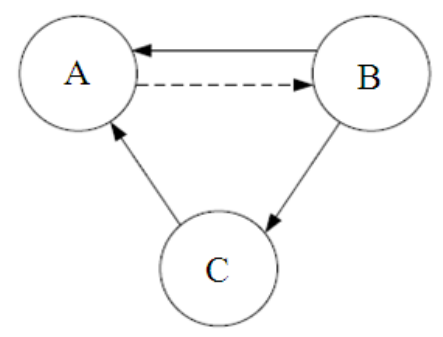

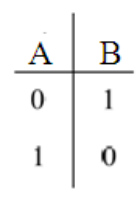
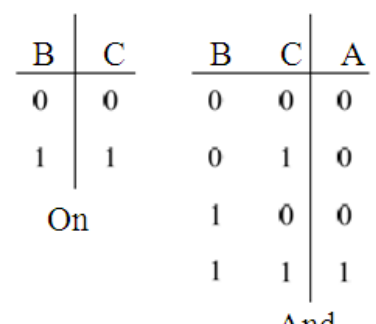

And

Fig. 1. The Boolean network of Example 1 and the truth tables of its excitations

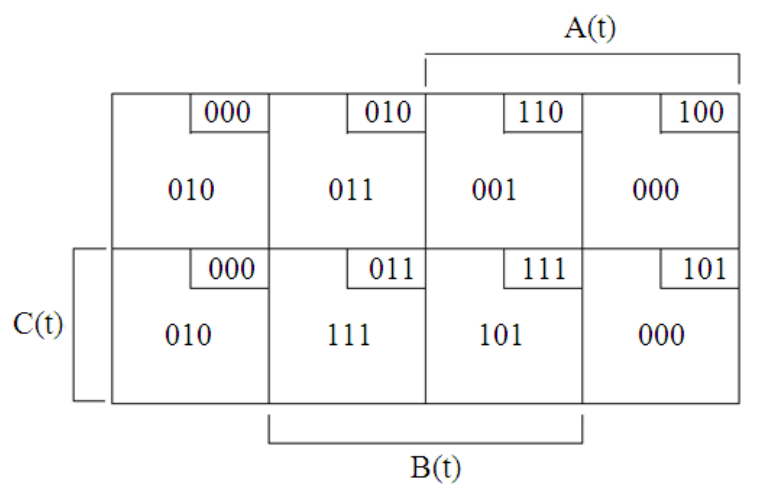

Fig. 2. A karnaugh-map representations of the next-next state functions $\{\mathrm{A}(\mathrm{t}+1) \mathrm{B}(\mathrm{t}+1) \mathrm{C}(\mathrm{t}+1)\}$ of the network in Example 1

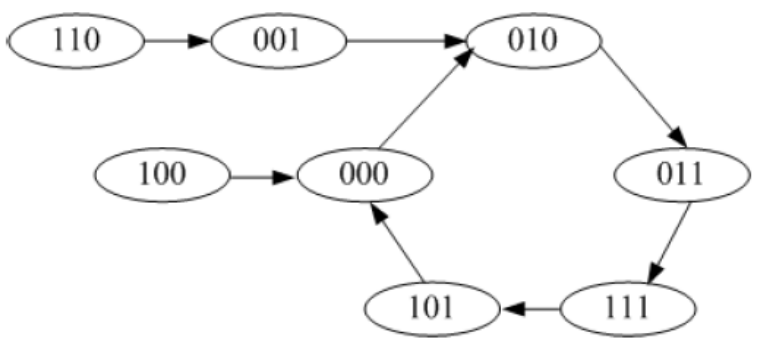

Fig. 3. The state diagram or map of all possible trajectories of the states $\{\mathrm{A}(\mathrm{t}) \mathrm{B}(\mathrm{t}) \mathrm{C}(\mathrm{t})\}$ of Example 1
While (15) is a novel condition, (16) is just an iteration of (13).

Further increments in $\mathrm{t}$ produce (with the invocation of (13) and (8)) Equation (17):

$$
\begin{aligned}
\mathrm{A}(\mathrm{t}+5)= & (1+\mathrm{A}(\mathrm{t}+3))(1+\mathrm{A}(\mathrm{t}+2)) \\
= & (1+(1+\mathrm{A}(\mathrm{t}))(1+\mathrm{A}(\mathrm{t}+1))) \\
& (1+\mathrm{A}(\mathrm{t}+2)) \\
= & (1+\mathrm{A}(\mathrm{t}+2))+(1+\mathrm{A}(\mathrm{t})) \\
& (1+\mathrm{A}(\mathrm{t}+1))(1+\mathrm{A}(\mathrm{t}+2)) \\
= & 1+\mathrm{A}(\mathrm{t}+2)+1+\mathrm{A}(\mathrm{t})+\mathrm{A}(\mathrm{t}+1) \\
+ & \mathrm{A}(\mathrm{t}+2)+\mathrm{A}(\mathrm{t}) \mathrm{A}(\mathrm{t}+2) \\
+ & \mathrm{A}(\mathrm{t}) \mathrm{A}(\mathrm{t}+1)+\mathrm{A}(\mathrm{t}+1) \\
& \mathrm{A}(\mathrm{t}+2)+\mathrm{A}(\mathrm{t}) \mathrm{A}(\mathrm{t}+1) \mathrm{A}(\mathrm{t}+2) \\
= & \mathrm{A}(\mathrm{t})+\mathrm{A}(\mathrm{t}+1)+\mathrm{A}(\mathrm{t}) \mathrm{A}(\mathrm{t}+1) \\
+ & \mathrm{A}(\mathrm{t}+1) \mathrm{A}(\mathrm{t}+2) \\
= & \mathrm{A}(\mathrm{t})+\mathrm{A}(\mathrm{t}+1)[1+\mathrm{A}(\mathrm{t})+\mathrm{A}(\mathrm{t}+2)] \\
= & \mathrm{A}(\mathrm{t})+\mathrm{B}(\mathrm{t}) \mathrm{C}(\mathrm{t})[\quad \mathrm{B}(\mathrm{t}+1) \\
= & \mathrm{A}(\mathrm{t})+\mathrm{B}(\mathrm{t})[1+\mathrm{B}(\mathrm{t})] \mathrm{t}(\mathrm{t}) \mathrm{B}(\mathrm{t}+1) \mathrm{B}(\mathrm{t})] \\
= & \mathrm{A}(\mathrm{t})+0=\mathrm{A}(\mathrm{t})
\end{aligned}
$$

which is a reduced scalar equation for A. It is the minimal such equation, since it has no preceding version $\left(\right.$ Here $\left.s_{a}=0\right)$.

Similarly, we increment (11b) to obtain Equation (18):

$\mathrm{B}(\mathrm{t}+2)=1+\mathrm{A}(\mathrm{t}+1)=1+\mathrm{B}(\mathrm{t}) \mathrm{C}(\mathrm{t})$

which can be used to produce an orthogonality relation Equation (19):

$\overline{\mathrm{B}}(\mathrm{t}+2) \overline{\mathrm{B}}(\mathrm{t})=0$

Further updating of t produces Equation (20 and 21):

$\mathrm{B}(\mathrm{t}+3)=1+\mathrm{B}(\mathrm{t}+1) \mathrm{C}(\mathrm{t}+1)$

$=1+\mathrm{B}(\mathrm{t}+1) \mathrm{B}(\mathrm{t})$

which is the scalar equation for B. An orthogonality relation involving three instances can be obtained from (20) Equation (22):

$$
\mathrm{B}(\mathrm{t}) \mathrm{B}(\mathrm{t}+1) \mathrm{B}(\mathrm{t}+3)=0
$$

Tow further increments in t produce Equation (23):

$$
\begin{aligned}
B(t+5) & =1+B(t+3) B(t+2) \\
& =1+[1+B(t+1) B(t)] B(t+2)
\end{aligned}
$$


which does not reduce to $\mathrm{B}(\mathrm{t})$ in general. A further increment in $\mathrm{t}$ results in Equation (24):

$$
\begin{gathered}
\mathrm{B}(\mathrm{t}+6)=1+[1+\mathrm{B}(\mathrm{t}+2) \mathrm{B}(\mathrm{t}+1)] \\
{[1+\mathrm{B}(\mathrm{t}) \mathrm{B}(\mathrm{t}+1)]} \\
=\mathrm{B}(\mathrm{t}+1) \\
{[\mathrm{B}(\mathrm{t}+2)+\mathrm{B}(\mathrm{t})+\mathrm{B}(\mathrm{t}) \mathrm{B}(\mathrm{t}+2)]} \\
\mathrm{B}(\mathrm{t}+6)=\mathrm{B}(\mathrm{t}+1)
\end{gathered}
$$

which is the reduced scalar equation for B. It resulted from Equation (25):

$$
\begin{aligned}
\mathrm{B}(\mathrm{t}+2) & +\mathrm{B}(\mathrm{t})+\mathrm{B}(\mathrm{t}) \mathrm{B}(\mathrm{t}+2) \\
& =1+\mathrm{B}(\mathrm{t}) \mathrm{C}(\mathrm{t})+\mathrm{B}(\mathrm{t})+\mathrm{B}(\mathrm{t})+\mathrm{B}(\mathrm{t}) \mathrm{C}(\mathrm{t}) \\
& =1
\end{aligned}
$$

Now, we increment (11c) to get Equation (26 to 28):

$$
\begin{aligned}
\mathrm{C}(\mathrm{t}+1) & =\mathrm{B}(\mathrm{t}) \\
\mathrm{C}(\mathrm{t}+2) & =\mathrm{B}(\mathrm{t}+1)=1+\mathrm{A}(\mathrm{t}) \\
\mathrm{C}(\mathrm{t}+3) & =1+\mathrm{A}(\mathrm{t}+1)=1+\mathrm{B}(\mathrm{t}) \mathrm{C}(\mathrm{t}) \\
& =1+\mathrm{C}(\mathrm{t}+1) \mathrm{C}(\mathrm{t})
\end{aligned}
$$

which is the scalar equation for C. It is similar to that for B (Equation (21)). However, as will turn out shortly, the reduced scalar equations for $\mathrm{B}$ and $\mathrm{C}$ are not the same. The variable $\mathrm{C}$ has a 3-instance orthogonality relation Equation (29):

$$
\mathrm{C}(\mathrm{t}) \mathrm{C}(\mathrm{t}+1) \mathrm{C}(\mathrm{t}+3)=0
$$

Further increments in t produce Equation (30 to 32):

$$
\begin{aligned}
\mathrm{C}(\mathrm{t}+4)= & 1+\mathrm{C}(\mathrm{t}+2) \mathrm{C}(\mathrm{t}+1) \\
\mathrm{C}(\mathrm{t}+5)= & 1+\mathrm{C}(\mathrm{t}+3) \mathrm{C}(\mathrm{t}+2) \\
= & 1+[1+\mathrm{C}(\mathrm{t}) \mathrm{C}(\mathrm{t}+1)] \mathrm{C}(\mathrm{t}+2) \\
= & 1+\mathrm{C}(\mathrm{t}+2)+\mathrm{C}(\mathrm{t}) \mathrm{C}(\mathrm{t}+1) \mathrm{C}(\mathrm{t}+2) \\
\mathrm{C}(\mathrm{t}+6)= & 1+[1+\mathrm{C}(\mathrm{t}+1) \mathrm{C}(\mathrm{t}+2)] \mathrm{C}(\mathrm{t}+3) \\
= & 1+[1+\mathrm{C}(\mathrm{t}+1) \mathrm{C}(\mathrm{t}+2)] \\
& \quad[1+\mathrm{C}(\mathrm{t}) \mathrm{C}(\mathrm{t}+2)] \\
= & 1+1+\mathrm{C}(\mathrm{t}+1) \mathrm{C}(\mathrm{t}+2) \\
& \quad+\mathrm{C}(\mathrm{t}) \mathrm{C}(\mathrm{t}+1) \\
& \quad+\mathrm{C}(\mathrm{t}) \mathrm{C}(\mathrm{t}+1) \mathrm{C}(\mathrm{t}+2) \\
= & \mathrm{C}(\mathrm{t}+1)[\mathrm{C}(\mathrm{t})+\mathrm{C}(\mathrm{t}+2)+\mathrm{C}(\mathrm{t}) \mathrm{C}(\mathrm{t}+2)] \\
= & \mathrm{C}(\mathrm{t}+1)[\mathrm{C}(\mathrm{t}) \mathrm{VC}(\mathrm{t}+2)]
\end{aligned}
$$

Note that the candidate reduced scalar equation: $\mathrm{C}(\mathrm{t}+6) \stackrel{?}{=} \mathrm{S}(\mathrm{t}+1)$ is valid if $\{\mathrm{C}(\mathrm{t}) \mathrm{VC}(\mathrm{t}+2)=1\}$, i.e., if $\{\overline{\mathrm{C}}(\mathrm{t}) \overline{\mathrm{C}}(\mathrm{t}+2=0$, i.e., if CtAt $=0$, which is not necessarily the case.

The next updated equation Equation (33):

$\mathrm{C}(\mathrm{t}+7)=\mathrm{C}(\mathrm{t}+2)[\mathrm{C}(\mathrm{t}+1) \mathrm{VC}(\mathrm{t}+3)]$

suggest that the next candidate reduced scalar equation:

$\mathrm{C}(\mathrm{t}+7)=\mathrm{C}(\mathrm{t}+2)$

is true provided $\{\mathrm{C}(\mathrm{t}+1) \mathrm{VC}(\mathrm{t}+3)=1\}$, i.e., provided $\{\overline{\mathrm{C}}(\mathrm{t}+1) \mathrm{A}(\mathrm{t}+1)=0\}$, which is true, since $\{\overline{\mathrm{C}}(\mathrm{t}+1)=\overline{\mathrm{B}}(\mathrm{t})\}$ and $\{\mathrm{A}(\mathrm{t}+1)=\mathrm{B}(\mathrm{t}) \mathrm{C}(\mathrm{t})\}$. Hence $(33)$ is the reduced scalar equation for $\mathrm{C}$.

Contrary to a claim made in [10, p. 350], the reduced scalar equations for the variables $\mathrm{A}, \mathrm{B}$ and $\mathrm{C}$ (Equation (17), (24) and (34)) are not the same. Each of these equations is satisfied when any of the eight states in the state diagram in Fig. 1 is taken as the basis or $t$ state. When combined together, they indicate that $\mathrm{X}(\mathrm{t}+7)=$ $\mathrm{X}(\mathrm{t}+2)$, where $\mathrm{X}$ stands for $\mathrm{A}, \mathrm{B}$, or $\mathrm{C}$. This means that the maximum transient in the state diagram is of length two 5. This allows the possibility of cycles of length equal to divisors of 5 (i.e., 1or 5). It can be shown that the network has no fixed points (cycle of length 1) $\{$ the system $A(t)=$ $\mathrm{B}(\mathrm{t}) \mathrm{C}(\mathrm{t}), \mathrm{B}(\mathrm{t})=1+\mathrm{A}(\mathrm{t}), \mathrm{C}(\mathrm{t})=\mathrm{B}(\mathrm{t})$ is obviously inconsistent and has no solution \}. The network could have only a single cycle of length 5 since its total number of states is 8 . Since the network has a single cycle of length 5 and one transient of length 2 , it must have a second transient of length 1 . Here, we used very simple reasoning to account for all network states, but this will not suffice when dealing with large complicated networks.

\subsection{Example 2}

Figure 4 presents the Boolean model of cell growth, differentiation and apoptosis (programmed cell death) introduced by Huang and Ingber (2000) and solved by Farrow et al. (2004) and by Cheng et al. (2011). For simplicity, the 11 nodes of the network are represented by the symbols A through K. The connectivity graph and logic tables are shown in Fig. 4 (IMP = "IMPLICATION", NIF = "NOT IF", which is also called "INHIBIT" and represents the complement of IMP, NAND = "NOT AND"). and the smallest common multiple of cycle length is 7-2 = 


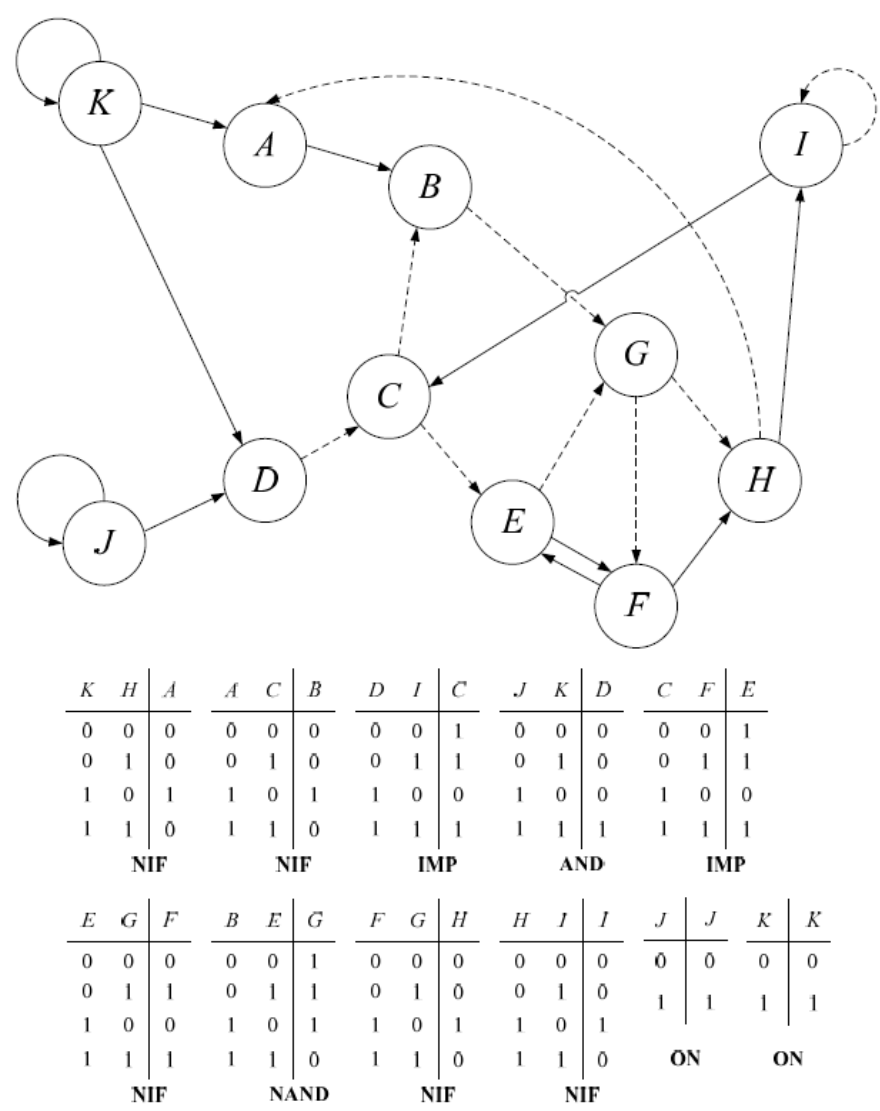

Fig. 4. The Boolean network of Example 2 and the truth tables of its excitations

Again, we use a solid arrow to indicate a "positive" effect, while a dotted arrow indicates a "negative" one. The network equations are as follows (Heidel et al., 2003; Huang and Ingber, 2000) Equation (35a to 35k):

$$
\begin{aligned}
& \mathrm{A}(\mathrm{t}+1)=\mathrm{K}(\mathrm{t})+\mathrm{K}(\mathrm{t}) \mathrm{H}(\mathrm{t}) \\
& \mathrm{B}(\mathrm{t}+1)=\mathrm{A}(\mathrm{t})+\mathrm{A}(\mathrm{t}) \mathrm{C}(\mathrm{t}) \\
& \mathrm{C}(\mathrm{t}+1)=1+\mathrm{D}(\mathrm{t})+\mathrm{D}(\mathrm{t}) \mathrm{I}(\mathrm{t}) \\
& \mathrm{D}(\mathrm{t}+1)=\mathrm{J}(\mathrm{t}) \mathrm{K}(\mathrm{t}) \\
& \mathrm{E}(\mathrm{t}+1)=1+\mathrm{C}(\mathrm{t})+\mathrm{C}(\mathrm{t}) \mathrm{F}(\mathrm{t}) \\
& \mathrm{F}(\mathrm{t}+1)=\mathrm{E}(\mathrm{t})+\mathrm{E}(\mathrm{t}) \mathrm{G}(\mathrm{t}) \\
& \mathrm{G}(\mathrm{t}+1)=1+\mathrm{B}(\mathrm{t}) \mathrm{E}(\mathrm{t})
\end{aligned}
$$

$$
\begin{aligned}
& H(t+1)=F(t)+F(t) G(t) \\
& I(t+1)=H(t)+H(t) I(t)=H(t) \bar{I}(t) \\
& J(t+1)=J(t) \\
& K(t+1)=K(t)
\end{aligned}
$$

Huang and Ingber (2000) have shown that a nontrivial growth attractor exists, assuming that the growth factor (node $\mathrm{k}$ ) and cell spreading (node $\mathrm{J}$ ) are both $\mathrm{ON}$, which means that node $\mathrm{D}$ is also $\mathrm{ON}(\mathrm{k}(\mathrm{t})=$ $\mathrm{J}(\mathrm{t})=\mathrm{Dt}=1$. In this case Equation (35a' and $\left.35 \mathrm{~b}^{\prime}\right)$ :

$$
\begin{aligned}
& \mathrm{A}(\mathrm{t}+1)=1+\mathrm{H}(\mathrm{t})=\overline{\mathrm{H}}(\mathrm{t}) \\
& \mathrm{C}(\mathrm{t}+1)=\mathrm{I}(\mathrm{t})
\end{aligned}
$$

Incrementing the value of $\mathrm{t}$ in (35i), one obtains Equation (36): 


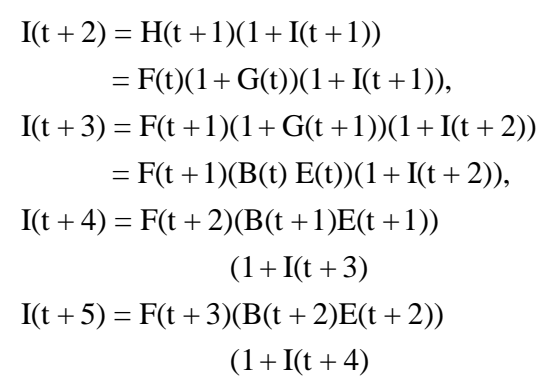

$$
\begin{aligned}
& I(t+1) I(t+5)=0 \\
& I(t+4) I(t+5)=0
\end{aligned}
$$

where Equation (37):

$$
\begin{aligned}
& \mathrm{F}(\mathrm{t}+2)= \mathrm{E}(\mathrm{t}+1)(1+\mathrm{G}(\mathrm{t}+1)) \\
&=(1+\mathrm{C}(\mathrm{t})+\mathrm{C}(\mathrm{t}) \mathrm{F}(\mathrm{t}))(\mathrm{B}(\mathrm{t}) \mathrm{E}(\mathrm{t})), \\
& \mathrm{F}(\mathrm{t}+3)=(1+\mathrm{C}(\mathrm{t}+1)+\mathrm{C}(\mathrm{t}+1) \mathrm{F}(\mathrm{t}+1)) \\
&(\mathrm{B}(\mathrm{t}+1) \mathrm{E}(\mathrm{t}+1)), \\
&(1+\mathrm{I}(\mathrm{t})+\mathrm{I}(\mathrm{t}) \mathrm{F}(\mathrm{t}+1)) \\
&(\mathrm{B}(\mathrm{t}+1) \mathrm{E}(\mathrm{t}+1)) \\
& \mathrm{F}(\mathrm{t}+4)=(1+\mathrm{I}(\mathrm{t}+1)+\mathrm{I}(\mathrm{t}+1) \mathrm{F}(\mathrm{t}+2)) \\
&(\mathrm{B}(\mathrm{t}+2) \mathrm{E}(\mathrm{t}+2))
\end{aligned}
$$

Now Equation (38):

$$
\begin{aligned}
& \mathrm{B}(\mathrm{t}+2) \mathrm{E}(\mathrm{t}+2)=\mathrm{A}(\mathrm{t}+1)(1+\mathrm{C}(\mathrm{t}+1)) \\
&(1+\mathrm{C}(\mathrm{t}+1)+\mathrm{C}(\mathrm{t}+1) \mathrm{F}(\mathrm{t}+1))=\mathrm{A}(\mathrm{t}+1) \\
& {\left[\begin{array}{l}
(1+\mathrm{C}(\mathrm{t}+1) \\
+(1+\mathrm{C}(\mathrm{t}+1)) \mathrm{C}(\mathrm{t}+1) \mathrm{F}(\mathrm{t}+1)
\end{array}\right] } \\
&= \mathrm{A}(\mathrm{t}+1)[(1+\mathrm{C}(\mathrm{t}+1))+0] \\
&= \mathrm{A}(\mathrm{t}+1)(1+\mathrm{C}(\mathrm{t}+1))=\mathrm{B}(\mathrm{t}+2) \\
&=(1+\mathrm{H}(\mathrm{t}))(1+\mathrm{I}(\mathrm{t})) \\
&= 1+\mathrm{I}(\mathrm{t})+\mathrm{H}(\mathrm{t})+\mathrm{H}(\mathrm{t}) \mathrm{I}(\mathrm{t}) \\
&= 1+\mathrm{I}(\mathrm{t})+\mathrm{I}(\mathrm{t}+1) \\
&=(1+\mathrm{I}(\mathrm{t}))(1+\mathrm{I}(\mathrm{t}+1))
\end{aligned}
$$

In (38), we made use of the orthogonality relation Equation (39):

$\mathrm{I}(\mathrm{t}) \mathrm{I}(\mathrm{t}+1)=\mathrm{I}(\mathrm{t}) \mathrm{H}(\mathrm{t})+\mathrm{H}(\mathrm{t}) \mathrm{I}(\mathrm{t})=0$

Hence, we can substitute (38) in (36) to obtain Equation (40):

$$
\mathrm{I}(\mathrm{t}+5)=\mathrm{F}(\mathrm{t}+3)(1+\mathrm{I}(\mathrm{t}))(1+\mathrm{I}(\mathrm{t}+1))(1+\mathrm{I}(\mathrm{t}+4))
$$

If (40) is ANDed (multiplied) with $\mathrm{I}(\mathrm{t}), \mathrm{I}(\mathrm{t}+1$ and It+4) respectively, it yields the orthogonality relations Equation (41 to 43):

$$
\mathrm{I}(\mathrm{t}) \mathrm{I}(\mathrm{t}+5)=0
$$

Of which (41) and (42) are new orthogonality relations, while (43) is simply a consequent or an updating of (39). We now substitute (38) in (37) to obtain Equation (44):

$$
\begin{aligned}
\mathrm{F}(\mathrm{t}+4)= & (1+\mathrm{I}(\mathrm{t}+1)+\mathrm{I}(\mathrm{t}+1) \mathrm{F}(\mathrm{t}+2)) \\
& (1+\mathrm{I}(\mathrm{t}))(1+\mathrm{I}(\mathrm{t}+1)) \\
= & {[(1+\mathrm{I}(\mathrm{t}+1))+0](1+\mathrm{I}(\mathrm{t})) } \\
= & (1+\mathrm{I}(\mathrm{t}))(1+\mathrm{I}(\mathrm{t}+1)) \\
= & \mathrm{B}(\mathrm{t}+2)
\end{aligned}
$$

Now, we iterate (40) further to obtain Equation (45a and 45b):

$$
\begin{aligned}
& \mathrm{I}(\mathrm{t}+6)= F(\mathrm{t}+4)(1+\mathrm{I}(\mathrm{t}+1))(1+\mathrm{I}(\mathrm{t}+2))(1+\mathrm{I}(\mathrm{t}+5)) \\
&=(1+\mathrm{I}(\mathrm{t}))(1+\mathrm{I}(\mathrm{t}+1))(\mathrm{t}+\mathrm{I}(\mathrm{t}+1)) \\
&(1+\mathrm{I}(\mathrm{t}+2))(1+\mathrm{I}(\mathrm{t}+5) \\
&=(1+\mathrm{I}(\mathrm{t}))(1+\mathrm{I}(\mathrm{t}+1))(\mathrm{t}+\mathrm{I}(\mathrm{t}+2))(1+\mathrm{I}(\mathrm{t}+5) \\
&=\mathrm{I}(\mathrm{t}+1)+(1+\mathrm{I}(\mathrm{t}))(1+\mathrm{I}(\mathrm{t}+2))(1+\mathrm{I}(\mathrm{t}+5)) \\
&=1+\mathrm{I}(\mathrm{t})+\mathrm{I}(\mathrm{t}+1)+\mathrm{I}(\mathrm{t}+2)+\mathrm{I}(\mathrm{t}+5) \\
&+ \mathrm{I}(\mathrm{t}) \mathrm{I}(\mathrm{t}+2) \\
&+ \mathrm{I}(\mathrm{t}+2) \mathrm{I}(\mathrm{t}+5)
\end{aligned}
$$

where, we involved the orthogonality conditions (39), (41), (42) and an updated version of (39).

Equation (45) is the scalar equation for $\mathrm{I}(\mathrm{t})$ and has been derived earlier by Farrow et al. (2004). If (45) is ANDed (multiplied) with $\mathrm{I}(\mathrm{t}), \mathrm{I}(\mathrm{t}+1), \mathrm{I}(\mathrm{t}+2$ and $\mathrm{It}+5)$ respectively, it yields the relations:

$\mathrm{I}(\mathrm{t}) \mathrm{I}(\mathrm{t}+6)=0$

$\mathrm{I}(\mathrm{t}+1) \mathrm{I}(\mathrm{t}+6)=0$

$\mathrm{I}(\mathrm{t}+2) \mathrm{I}(\mathrm{t}+6)=0$

$I(t+5) I(t+6)=0$

Of which only Equation (46a) is new, while Equation (46b), (42c) and (46d) are simply latter instances of Equation (41), (42) and (39), respectively. 
If we iterate (45) further, we obtain an expression of $\mathrm{I}(\mathrm{t}$ $+7)$, in which we substitute for $\mathrm{I}(\mathrm{t}+6)$ from $(45)$ to obtain Equation (47):

$$
\begin{array}{r}
\mathrm{I}(\mathrm{t}+7)=(1+\mathrm{I}(\mathrm{t}+1))(1+\mathrm{I}(\mathrm{t}+2)) \\
(1+\mathrm{I}(\mathrm{t}+3))(1+\mathrm{I}(\mathrm{t}+6)) \\
=(1+\mathrm{I}(\mathrm{t}+1))(1+\mathrm{I}(\mathrm{t}+2)) \\
(1+\mathrm{I}(\mathrm{t}+3))+(1+\mathrm{I}(\mathrm{t})) \\
(1+\mathrm{I}(\mathrm{t}+1))(1+\mathrm{I}(\mathrm{t}+2)) \\
(1+\mathrm{I}(\mathrm{t}+3))(1+\mathrm{I}(\mathrm{t}+5)) . \\
=(1+\mathrm{I}(\mathrm{t}+1))(1+\mathrm{I}(\mathrm{t}+2)) \\
(1+\mathrm{I}(\mathrm{t}+3)) \\
{\left[\begin{array}{l}
1+1+\mathrm{I}(\mathrm{t})+\mathrm{I}(\mathrm{t}+5) \\
+\mathrm{I}(\mathrm{t}) \mathrm{I}(\mathrm{t}+5)
\end{array}\right]} \\
=(\mathrm{I}(\mathrm{t})+\mathrm{I}(\mathrm{t}+5))(1+\mathrm{I}(\mathrm{t}+1)) \\
(1+\mathrm{I}(\mathrm{t}+2))(1+\mathrm{I}(\mathrm{t}+3))
\end{array}
$$

where, the orthogonality condition (41) is invoked. Note that in general, there is no orthogonality between $\mathrm{I}(\mathrm{t}+4)$ and $\mathrm{I}(\mathrm{t}+7)$, namely Equation (48):

$$
\begin{aligned}
\mathrm{I}(\mathrm{t}+4) \mathrm{I}(\mathrm{t}+7)=(\mathrm{I}(\mathrm{t}) \mathrm{I}(\mathrm{t}+4)+\mathrm{I}(\mathrm{t}+4) \mathrm{I}(\mathrm{t}+5)) \\
(1+\mathrm{I}(\mathrm{t}+1)) \\
(1+\mathrm{I}(\mathrm{t}+2)) \\
\quad\left(\begin{array}{l}
\mathrm{I}(\mathrm{t}+4) \\
+\mathrm{I}(\mathrm{t}+3) \mathrm{I}(\mathrm{t}+4)
\end{array}\right) \\
=\mathrm{I}(\mathrm{t}) \mathrm{I}(\mathrm{t}+4)(1+\mathrm{I}(\mathrm{t}+1)) 1+\mathrm{I}(\mathrm{t}+2)) \\
=\mathrm{I}(\mathrm{t}) \mathrm{I}(\mathrm{t}+4)(1+\mathrm{I}(\mathrm{t}+2)) \\
\neq 0
\end{aligned}
$$

In (48), we utilized the orthogonality condition (39) three times.

Now, we iterate (47) further and use (45c) to obtain:

$$
\begin{array}{r}
\mathrm{I}(\mathrm{t}+8)=(\mathrm{I}(\mathrm{t}+1)+\mathrm{I}(\mathrm{t}+6))(1+\mathrm{I}(\mathrm{t}+2)) \\
(1+\mathrm{I}(\mathrm{t}+3))(1+\mathrm{I}(\mathrm{t}+4)) \\
=(1+\mathrm{I})(\mathrm{t}))(1+2))(1+\mathrm{I}(\mathrm{t}+3)) \\
(1+\mathrm{I}(\mathrm{t}+4))(1+\mathrm{I}(\mathrm{t}+5))
\end{array}
$$

Equation (49) is a much simpler expression for $\mathrm{I}(\mathrm{t}+8)$ than the one in Farrow et al. (2004). It supplies a new orthogonality relation Equation (50):

$$
\mathrm{I}(\mathrm{t}+5) \mathrm{I}(\mathrm{t}+8)=0
$$

Table 1 summarizes the orthogonality relations obtained so far. Clearly, some of them start from the outset, while some start after some delay.

Further iteration yields Equation (51):

$$
\begin{gathered}
\mathrm{I}(\mathrm{t}+9)=(1+\mathrm{I}(\mathrm{t}+1))(1+\mathrm{I}(\mathrm{t}+3)) \\
(1+\mathrm{I}(\mathrm{t}+4))(1+\mathrm{I}(\mathrm{t}+5))(1+\mathrm{I}(\mathrm{t}+6) \\
=(1+\mathrm{I}(\mathrm{t}+1))(1+\mathrm{I}(\mathrm{t}+3) \\
(1+\mathrm{I}(\mathrm{t}+4))(1+\mathrm{I}(\mathrm{t}+5))+(1+\mathrm{I}(\mathrm{t}))(1+\mathrm{I}(\mathrm{t}+1)) \\
(1+\mathrm{I}(\mathrm{t}+2))(1+\mathrm{I}(\mathrm{t}+3))(1+\mathrm{I}(\mathrm{t}+4))(1+\mathrm{I}(\mathrm{t}+5)) \\
=(1+\mathrm{I}(\mathrm{t}+1))(1+\mathrm{I}(\mathrm{t}+3))(1+\mathrm{I}(\mathrm{t}+4))(1+\mathrm{I}(\mathrm{t}+5)) \\
\quad[\mathrm{I}(\mathrm{t})+\mathrm{I}(\mathrm{t}+2)+\mathrm{I}(\mathrm{t}) \mathrm{I}(\mathrm{t}+2)] \\
\mathrm{I}(\mathrm{t}+10)=(1+\mathrm{I}(\mathrm{t}+2))(1+\mathrm{I}(\mathrm{t}+4)) \\
(1+\mathrm{I}(\mathrm{t}+5))(1+\mathrm{I}(\mathrm{t}+6)) \\
{\left[\begin{array}{l}
\mathrm{I}(\mathrm{t}+1)+\mathrm{I}(\mathrm{t}+3) \\
+\mathrm{I}(\mathrm{t}+1) \mathrm{I}(\mathrm{t}+3)
\end{array}\right]} \\
=[(1+\mathrm{I}(\mathrm{t}+2))(1+\mathrm{I}(\mathrm{t}+4)) \\
(1+\mathrm{I}(\mathrm{t}+5))+(1+\mathrm{I}(\mathrm{t})) \\
(1+\mathrm{I}(\mathrm{t}+1))(1+\mathrm{I}(\mathrm{t}+2)) \\
(1+\mathrm{I}(\mathrm{t}+4))(1+\mathrm{I}(\mathrm{t}+5))] \\
{\left[\begin{array}{l}
\mathrm{I}(\mathrm{t}+1)+\mathrm{I}(\mathrm{t}+3)] \\
+\mathrm{I}(\mathrm{t}+1) \mathrm{I}(\mathrm{t}+3)
\end{array}\right]} \\
=(1+\mathrm{I}(\mathrm{t}+2))(1+\mathrm{I}(\mathrm{t}+4)) \\
(1+\mathrm{I})(\mathrm{t}+5)[\mathrm{I}(\mathrm{t})+\mathrm{I}(\mathrm{t}+1)] \\
{\left[\begin{array}{l}
\mathrm{I}(\mathrm{t}+1)+\mathrm{I}(\mathrm{t}+3)] \\
+\mathrm{I}(\mathrm{t}+1) \mathrm{I}(\mathrm{t}+3)
\end{array}\right]} \\
=(1+\mathrm{I}(\mathrm{t}+2))(1+\mathrm{I}(\mathrm{t}+4)) \\
(1+\mathrm{I}(\mathrm{t}+5)) \\
{[\mathrm{I}(\mathrm{t}) \mathrm{I}(\mathrm{t}+3)+\mathrm{I}(\mathrm{t}+1)]}
\end{gathered}
$$

where, the condition (39) and the identity (4) are used. Note that Equation (52a):

$$
\begin{gathered}
\mathrm{I}(\mathrm{t}) \mathrm{I}(\mathrm{t}+3)(1+\mathrm{I}(\mathrm{t}+2))(1+\mathrm{I}(\mathrm{t}+4))(1+\mathrm{It}+5) \\
=(\mathrm{I}(\mathrm{t}+3)+\mathrm{I}(\mathrm{t}+2) \mathrm{I}(\mathrm{t}+3)) \\
(\mathrm{I}(\mathrm{t}+3)+\mathrm{I}(\mathrm{t}+3) \mathrm{I}(\mathrm{t}+4)) \\
(\mathrm{I}(\mathrm{t})+\mathrm{I}(\mathrm{t}) \mathrm{I}(\mathrm{t}+5)) \\
=(\mathrm{I}(\mathrm{t}+3)+0)(\mathrm{I}(\mathrm{t}+3)+0)(\mathrm{I}(\mathrm{t})+0) \\
=\mathrm{I}(\mathrm{t}) \mathrm{I}(\mathrm{t}+3) \\
(\mathrm{I}(\mathrm{t}+1)(1+\mathrm{I}(\mathrm{t}+2))(1+\mathrm{I}(\mathrm{t}+4))(1+\mathrm{I}(\mathrm{t}+5)) \\
=(\mathrm{I}(\mathrm{t}+1)+\mathrm{I}(\mathrm{t}+1) \mathrm{I}(\mathrm{t}+2)) \\
\quad(1+\mathrm{I}(\mathrm{t}+4)) \\
(\mathrm{I}(\mathrm{t}+1)+\mathrm{I}(\mathrm{t}+1) \mathrm{I}(\mathrm{t}+5)) \\
=(\mathrm{I}(\mathrm{t}+1)+0)(1+\mathrm{I}(\mathrm{t}+4))(\mathrm{I}(\mathrm{t}+1)+0) \\
=\mathrm{I}(\mathrm{t}+1)+1(\mathrm{t}+1) \mathrm{I}(\mathrm{t}+4)
\end{gathered}
$$


Table 1. Set of orthogonality relations for I $(\mathrm{t})$

\begin{tabular}{lll}
\hline $\begin{array}{l}\text { Time } \\
\text { distance }\end{array}$ & $\begin{array}{l}\text { First instance of an } \\
\text { orthogonality relation }\end{array}$ & $\begin{array}{l}\text { Equation } \\
\text { number }\end{array}$ \\
\hline 1 & $\mathrm{I}(\mathrm{t}) \mathrm{I}(\mathrm{t}+1)=0$ & $(39)$ \\
2 & - & - \\
3 & $\mathrm{I}(\mathrm{t}+5) \mathrm{I}(\mathrm{t}+8)=0$ & $(50)$ \\
4 & $\mathrm{I}(\mathrm{t}+1) \mathrm{I}(\mathrm{t}+5)=0$ & $(42)$ \\
5 & $\mathrm{I}(\mathrm{t}) \mathrm{I}(\mathrm{t}+5)=0$ & $(41)$ \\
6 & $\mathrm{I}(\mathrm{t}) \mathrm{I}(\mathrm{t}+6)=0$ & $(46)$ \\
\hline
\end{tabular}

Hence, expression (51) for $\mathrm{I}(\mathrm{t}+10)$ simplifies to Equation (52b-e-54):

$$
\begin{aligned}
& I(t+10)=I(t+1)+I(t) I(t+3)+I(t+1) I(t+4) \\
& I(t+11)=I(t+2)+I(t+1)+I(t+4)+I(t+2) I(t+5) \\
& I(t+12)=I(t+3)+I(t+2)+I(t+5)+I(t+3) I(t+6) \\
& I(t+13)=I(t+4)+I(t+3)+I(t+6)+I(t+4) I(t+7) \\
& I(t+14)=I(t+5)+I(t+4)+I(t+7)+I(t+5) I(t+8) \\
& =I(t+5)+I(t+4) I(t+7) \\
& I(t+14)
\end{aligned}
$$

where we made use of (50) and (48). We further update (53) and utilize (50) to obtain Equation (55):

$$
\begin{aligned}
& I(t+15)=I(t+6)+I(t+5) I(t+8) \\
& I(t+15)=I(t+6)
\end{aligned}
$$

The reduced scalar equation for $\mathrm{I}(\mathrm{t})$, Equation (56) is one time-period ahead of that given in (Farrow et al., 2004). Furthermore, contrary to assumptions made in (Farrow et al., 2004), other variables of the Boolean network do not necessarily have the same reduced scalar equation.

To obtain the reduced scalar equation for $E(t)$, we start from (35e) and substitute from (35b'), (35f), (35g), (38), (39), (50), (52a) and (53) as follows Equation (57):

$$
\begin{aligned}
& \mathrm{E}(\mathrm{t}+2)=1+\mathrm{C}(\mathrm{t}+1)(1+\mathrm{F}(\mathrm{t}+1))=1+\mathrm{I}(\mathrm{t})(1+\mathrm{E}(\mathrm{t}) \\
& +\mathrm{E}(\mathrm{t}) \mathrm{G}(\mathrm{t})) \mathrm{E}(\mathrm{t}+3)=1+\mathrm{I}(\mathrm{t}+1)(1+\mathrm{E}(\mathrm{t}+1)(1+1+\mathrm{B}(\mathrm{t}) \\
& \mathrm{E}(\mathrm{t})))=1+\mathrm{I}(\mathrm{t}+1)(1+\mathrm{E}(\mathrm{t}+1) \mathrm{E}(\mathrm{t}) \mathrm{B}(\mathrm{t})) \mathrm{E}(\mathrm{t}+5)=1+\mathrm{I}(\mathrm{t}+3) \\
& {\left[\begin{array}{l}
1+\mathrm{E}(\mathrm{t}+3) \\
\{\mathrm{E}(\mathrm{t}+2) \mathrm{B}(\mathrm{t}+2)\}
\end{array}\right]=1+\mathrm{I}(\mathrm{t}+3)\left[\begin{array}{l}
{\left[\begin{array}{l}
1+\mathrm{I}(\mathrm{t}+1) \\
1+\left[\begin{array}{l}
1+\mathrm{E}(\mathrm{t}+1) \\
\mathrm{E}(\mathrm{t}) \mathrm{B}(\mathrm{t})
\end{array}\right) \\
\left\{\begin{array}{l}
(1+\mathrm{I}(\mathrm{t})) \\
(1+\mathrm{I}(\mathrm{t}+1))
\end{array}\right\}
\end{array}\right]}
\end{array}\right.}
\end{aligned}
$$

$$
\begin{aligned}
& =1+\mathrm{I}(\mathrm{t}+3)[1+(1+\mathrm{I}(\mathrm{t}))(1+\mathrm{I}(\mathrm{t}+1))] \\
& =1+\mathrm{I}(\mathrm{t}+3)(\mathrm{I}(\mathrm{t})+\mathrm{I}(\mathrm{t}+1)) \\
& \mathrm{E}(\mathrm{t}+10)=1+\mathrm{I}(\mathrm{t}+8)(\mathrm{I}(\mathrm{t}+5)+\mathrm{I}(\mathrm{t}+6)) \\
& =1+\mathrm{I}(\mathrm{t}+6) \mathrm{I}(\mathrm{t}+8) \\
& \mathrm{E}(\mathrm{t}+14)=1+\mathrm{I}(\mathrm{t}+10) \mathrm{I}(\mathrm{t}+12) \\
& =1+\left[\begin{array}{l}
\mathrm{I}(\mathrm{t}+1)+\mathrm{I}(\mathrm{t}) \mathrm{I}(\mathrm{t}+3) \\
+\mathrm{I}(\mathrm{t}+1) \mathrm{I}(\mathrm{t}+4)
\end{array}\right] \\
& {\left[\begin{array}{l}
\mathrm{I}(\mathrm{t}+3)+\mathrm{I}(\mathrm{t}+2) \mathrm{I}(\mathrm{t}+5) \\
+\mathrm{I}(\mathrm{t}+3) \mathrm{I}(\mathrm{t}+6)
\end{array}\right]} \\
& =1+\mathrm{I}(\mathrm{t}+3)[\mathrm{I}(\mathrm{t})+\mathrm{I}(\mathrm{t}+1)]= \\
& \mathrm{E}(\mathrm{t}+5)
\end{aligned}
$$

In the last step towards (57), use has been made of the orthogonality relations (39), (41) and (42). Clearly, the reduced scalar Equation (56) and (57) for I and E, respectively, are not the same.

Now, we obtain the reduced scalar equation for $\mathrm{H}(\mathrm{t})$ from (35h) and making use of (35f), (35g), (38), (52c), (52d) and (52e) as follows Equation (58 and 59):

$$
\begin{aligned}
& \mathrm{H}(\mathrm{t}+2)= \mathrm{F}(\mathrm{t}+1)(1+\mathrm{G}(\mathrm{t}+1)) \\
&= \mathrm{E}(\mathrm{t})(1+\mathrm{G}(\mathrm{t}))(\mathrm{B}(\mathrm{t}) \mathrm{E}(\mathrm{t})) \\
& \mathrm{H}(\mathrm{t}+3)=\mathrm{E}(\mathrm{t}+1) \mathrm{B}(\mathrm{t}+1) \mathrm{B}(\mathrm{t}) \mathrm{E}(\mathrm{t}) \\
& \mathrm{H}(\mathrm{t}+5)=\mathrm{E}(\mathrm{t}+3) \mathrm{B}(\mathrm{t}+3) \mathrm{B}(\mathrm{t}+2) \mathrm{E}(\mathrm{t}+2) \\
&=(1+\mathrm{I}(\mathrm{t}))(1+\mathrm{I}(\mathrm{t}+1))(1+\mathrm{I}(\mathrm{t}+2)) \\
&=1+\mathrm{I}(\mathrm{t})) \mathrm{I}(\mathrm{t}+1))+\mathrm{I}(\mathrm{t}+2) \\
&+\mathrm{I}(\mathrm{t}) \mathrm{I}(\mathrm{t}+2) \\
& \mathrm{H}(\mathrm{t}+7)+1+\mathrm{I}(\mathrm{t}+2)+\mathrm{I}(\mathrm{t}+3)+\mathrm{I}(\mathrm{t}+4) \\
&+\mathrm{I}(\mathrm{t}+2) \mathrm{I}(\mathrm{t}+4) \\
& \mathrm{H}(\mathrm{t}+16)=1+\mathrm{I}(\mathrm{t}+11)+\mathrm{I}(\mathrm{t}+12) \\
&+(\mathrm{I}(\mathrm{t}+13)+\mathrm{I}(\mathrm{t}+11) \mathrm{I}(\mathrm{t}+13) \\
&=1+\mathrm{I}(\mathrm{t}+2)+\mathrm{I}(\mathrm{t}+1) \mathrm{I}(\mathrm{t}+4) \\
&+ \mathrm{I}(\mathrm{t}+2) \mathrm{I}(\mathrm{t}+5) \\
&+\mathrm{I}(\mathrm{t}+3)+\mathrm{I}(\mathrm{t}+2) \mathrm{I}(\mathrm{t}+5) \\
&+\mathrm{I}(\mathrm{t}+3) \mathrm{I}(\mathrm{t}+6) \\
&+\mathrm{I}(\mathrm{t}+4)+\mathrm{I}(\mathrm{t}+3) \mathrm{I}(\mathrm{t}+6) \\
&+\mathrm{I}(\mathrm{t}+4) \mathrm{I}(\mathrm{t}+7) \\
&+\mathrm{I}(\mathrm{t}+2) \mathrm{I}(\mathrm{t}+4) \\
&+\mathrm{I}(\mathrm{t}+1) \mathrm{I}(\mathrm{t}+4) \\
&=1+ \mathrm{I}(\mathrm{t}+2)+\mathrm{I}(\mathrm{t}+3)+\mathrm{I}(\mathrm{t}+4) \\
&+\mathrm{I}(\mathrm{t}+2) \mathrm{I}(\mathrm{t}+4) \\
&+\mathrm{I}(\mathrm{t}+4)(\mathrm{I}(\mathrm{t}+7) \\
&= \mathrm{H}(\mathrm{t}+7)+\mathrm{I}(\mathrm{t}+4) \mathrm{I}(\mathrm{t}+7) \\
& \neq \mathrm{H}(\mathrm{t}+7)
\end{aligned}
$$


$H(t+17)=H(t+8)+I(t+5) I(t+8)=H(t+8)$

Equation (60), is the reduced scalar equation for $\mathrm{H}$. It resulted from the orthogonality relation (50), while (59) resulted from the denial of orthogonality in Equation (48).

To obtain the reduced scalar equation for $\mathrm{B}(\mathrm{t})$, we start from (38) and utilize (53), (56), (48) and (50) as follows Equation (61):

$$
\begin{aligned}
& \mathrm{B}(\mathrm{t}+2)= \\
& =1+\mathrm{I}(\mathrm{t})+\mathrm{I}(\mathrm{t})+\mathrm{I}(\mathrm{t}+1) \\
& \mathrm{B}(\mathrm{t}+7)=1+\mathrm{I}(\mathrm{t}+5)+\mathrm{I}(\mathrm{t}+6) \\
& \mathrm{B}(\mathrm{t}+16)=1+\mathrm{I}(\mathrm{t}+14)+\mathrm{I}(\mathrm{t}+15) \\
& =1+\mathrm{I}(\mathrm{t}+5)=+\mathrm{I}(\mathrm{t}+4)+\mathrm{I}(\mathrm{t}+7)+\mathrm{I}(\mathrm{t}+6) \\
& =\mathrm{B}(\mathrm{t}+7)=+\mathrm{I}(\mathrm{t}+4)+\mathrm{I}(\mathrm{t}+7) \neq \mathrm{B}(\mathrm{t}+7)
\end{aligned}
$$

$\mathrm{B}(\mathrm{t}+17)=\mathrm{B}(\mathrm{t}+8)+\mathrm{I}(\mathrm{t}+5) \mathrm{I}(\mathrm{t}+8)=\mathrm{B}(\mathrm{t}+8)$

The reduced scalar Equation (56), (57), (60) and (62) for I, E, H and B were verified by simulations. Reduced scalar equations for other variables of the network are Equation (63 to 66):

$$
\begin{aligned}
& \mathrm{A}+(\mathrm{t}+18)=\mathrm{A}(\mathrm{t}+9) \\
& \mathrm{C}+(\mathrm{t}+16)=\mathrm{C}(\mathrm{t}+7) \\
& \mathrm{F}+(\mathrm{t}+19)=\mathrm{F}(\mathrm{t}+10) \\
& \mathrm{G}+(\mathrm{t}+18)=\mathrm{G}(\mathrm{t}+9)
\end{aligned}
$$

These were proved with the aid of the earlier reduced scalar equations and were verified by simulation as well. In particular, (65) is deducible from (44) and (56). The maximum delay (transient period) $\mathrm{T}_{\mathrm{t}}$ encountered is 10 and takes place for $F(t)$ according to (65). This result is in agreement with that provided by the more elaborated matrix method of Cheng et al. (2011). The earlier scalar result in (Heidel et al., 2003), which is $T_{t} \leq 7$ is incorrect. Our update of the reduced scalar method refutes the possibility of existence of a discrepancy between scalar and matrix methods.

\subsection{Example 3}

The network in Fig. 5 is a simple case of an affine system (that has linear terms plus constant terms in the Reed-Müller expressions of its next state functions). Affine Boolean networks have been studied in great detail and their cyclic structure is completely understood in a general way (Milligan and Wilson, 1993; Wilson and Milligan, 1992), but the network of Fig. 5 has been analyzed by Heidel et al. (2003) and Rushdi and AlOtaibi (2008) via the scalar-equations technique.

The logic for this network is expressed by the following Equation (67a to f):

$$
\begin{aligned}
& \mathrm{A}(\mathrm{t}+1)=1+\mathrm{F}(\mathrm{t}) \\
& \mathrm{B}(\mathrm{t}+1)=\mathrm{A}(\mathrm{t}) \\
& \mathrm{C}(\mathrm{t}+1)=\mathrm{B}(\mathrm{t}) \\
& \mathrm{D}(\mathrm{t}+1)=\mathrm{C}(\mathrm{t}) \\
& \mathrm{E}(\mathrm{t}+1)=\mathrm{D}(\mathrm{t}) \\
& \mathrm{F}(\mathrm{t}+1)=\mathrm{E}(\mathrm{t})
\end{aligned}
$$

A scalar equation for $\mathrm{A}(\mathrm{t})$ is obtained as follows Equation (68a to e):

$$
\begin{aligned}
& \mathrm{A}(\mathrm{t}+2)=1+\mathrm{F}(\mathrm{t}+1)=1+\mathrm{E}(\mathrm{t}) \\
& \mathrm{A}(\mathrm{t}+3)=1+\mathrm{E}(\mathrm{t}+1)=1+\mathrm{D}(\mathrm{t}) \\
& \mathrm{A}(\mathrm{t}+4)=1+\mathrm{D}(\mathrm{t}+1)=1+\mathrm{C}(\mathrm{t}) \\
& \mathrm{A}(\mathrm{t}+5)=1+\mathrm{C}(\mathrm{t}+1)=1+\mathrm{B}(\mathrm{t}) \\
& \mathrm{A}(\mathrm{t}+6)=1+\mathrm{B}(\mathrm{t}+1)=1+\mathrm{A}(\mathrm{t})
\end{aligned}
$$

A reduced scalar equation can be derived by continuing as follows Equation (68f to $\mathrm{k}$ ):

$$
\begin{aligned}
& \mathrm{A}(\mathrm{t}+7)=1+\mathrm{A}(\mathrm{t}+1)=\mathrm{F}(\mathrm{t}) \\
& \mathrm{A}(\mathrm{t}+8)=\mathrm{F}(\mathrm{t}+1)=\mathrm{E}(\mathrm{t}) \\
& \mathrm{A}(\mathrm{t}+9)=\mathrm{E}(\mathrm{t}+1)=\mathrm{D}(\mathrm{t}) \\
& \mathrm{A}(\mathrm{t}+10)=\mathrm{D}(\mathrm{t}+1)=\mathrm{C}(\mathrm{t}) \\
& \mathrm{A}(\mathrm{t}+11)=\mathrm{C}(\mathrm{t}+1)=\mathrm{B}(\mathrm{t}) \\
& \mathrm{A}(\mathrm{t}+12)=\mathrm{B}(\mathrm{t}+1)=\mathrm{A}(\mathrm{t})
\end{aligned}
$$

Equation (68k) represents the reduced scalar equation for $A(t)$. This does not result from (68k) alone, since we need to show also that the condition Equation (69a):

$$
\mathrm{A}(\mathrm{t}+11) \stackrel{?}{=} \mathrm{A}(\mathrm{t})
$$




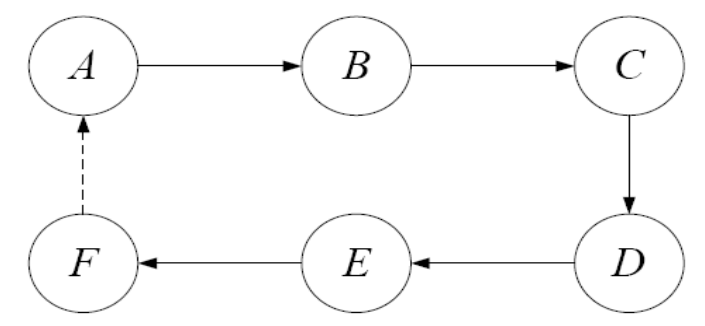

\begin{tabular}{c|ccccc|cc|cc|cc|c}
$A$ & $B$ & $B$ & $C$ & $C$ & $D$ & $D$ & $E$ & $E$ & $F$ & $F$ & $A$ \\
\hline 0 & 0 & 0 & 0 & 0 & 0 & 0 & 0 & 0 & 0 & & 1 & 0 \\
1 & 1 & 1 & 1 & 1 & 1 & 1 & 1 & 1 & 1 & 0 & 1 \\
ON & ON & ON & ON & ON & OFF
\end{tabular}

Fig. 5. The Boolean network of Example 3 and the truth tables of its excitations

Is not satisfied for all $\mathrm{t}$, which can be reduced by virtue of (67b) and (68j) to the condition that Equation (69b):

$$
\mathrm{B}(\mathrm{t}) \stackrel{?}{=} \mathrm{B}(\mathrm{t}+1)
$$

Is not satisfied for all t. Now if $(69 b)$ is true for all $t$, then $\mathrm{B}(\mathrm{t})$ remains constant and by virtue of equations $(67 \mathrm{c})$ to $(67 \mathrm{f})$ and $(67 \mathrm{a})$, also $\mathrm{C}(\mathrm{t}), \mathrm{D}(\mathrm{t}), \mathrm{E}(\mathrm{t}), \mathrm{F}(\mathrm{t})$ and $\mathrm{A}(\mathrm{t})$ remain constant. Hence, asserting $(69 \mathrm{~b})$ amounts to stucking the network at single particular states, i.e., making every network state a fixed point. This cannot be the case, since it contradicts (68e), which explicitly asserts that $\mathrm{A}(\mathrm{t})$ switches its value every six time units. This means that neither (69a) nor (69b) is true and hence $(68 \mathrm{k})$ is indeed the reduced scalar equation for $\mathrm{A}(\mathrm{t})$. Now, we seek a reduced scalar equation for $A(t)$ (since we might not be sure that it is the same as that of $A(t))$. From (68d), we have Equation (70 and 71):

$$
\begin{aligned}
& \mathrm{B}(\mathrm{t})=1+\mathrm{A}(\mathrm{t}+5) \\
& \mathrm{B}(\mathrm{t}+12)=1+\mathrm{A}(\mathrm{t}+17)=1+\mathrm{A}(\mathrm{t}+5)=\mathrm{B}(\mathrm{t})
\end{aligned}
$$

Equation (71) represents the reduced scalar equation for $\mathrm{B}(\mathrm{t})$ provided we can show that the condition Equation (72a):

$$
\mathrm{B}(\mathrm{t}+11) \stackrel{?}{=} \mathrm{B}(\mathrm{t})
$$

Is not satisfied for all t. By virtue of (67b), (68i) and $(67 \mathrm{c})$, condition $(72 \mathrm{a})$ reduces to the requirement that Equation (72b):

$$
\mathrm{C}(\mathrm{t}) \stackrel{?}{=} \mathrm{C}(\mathrm{t}+1)
$$

Be satisfied for all $t$, which is not the case according to our previous arguments. Note that B satisfies the same reduced scalar equation as A. Similarly, we can show that each of the other nodes C, D, E and F satisfies the same equation. This means that there is no transient state for this network $\left(n_{t}=0\right)$ and hence the length of the maxim transient trajectory is zero $\left(\mathrm{T}_{\mathrm{t}}=0\right)$ and there are cycles of period twelve. These cycles might include cycles of divisors of $12(1,2,3,4,6$ and 12), but thanks to $(68 \mathrm{e})$ we can negate the possibility of cycles of period six and its divisors (1, 2, 3 and 6). Therefore, the only possible cycle lengths are four and twelve.

The total number of states can be expressed as:

$2^{6}=64=4 n_{4}+12 n_{12}$

where $n_{4}$ is the number of period-four cycles and $n_{12}$ is the number of period-twelve cycles. Equation (73) can be rewritten as:

$\mathrm{n}_{4}+3 \mathrm{n}_{12}=16$

Equation (74) is a special Diophantine equation, i.e., an equation with integer coefficients for which integer solutions are sought (Andreescu and Andrica, 2009; Andreescu et al., 2010; Everest and Ward, 2005; Schroeder, 2009). This equation can be solved under the conditions that $\mathrm{n}_{4}$ and $\mathrm{n}_{12}$ are nonnegative integers as shown in Fig. 6. Equation (74) is represented a straight line in the twodimensional space of $n_{12}$ versus $n_{4}$, which initially suggests that (74) has an infinite number of solutions. However, both $\mathrm{n}_{4}$ and $\mathrm{n}_{12}$ are restricted to be nonnegative integers. This means that Equation (74) has a finite number of solutions. To stress the nonnegative integral nature of possible solutions of (74), the straight line representing (74) is drawn over a rectangular grid of lines, for which each of $n_{4}$ and $n_{12}$ has nonnegative integer values. 


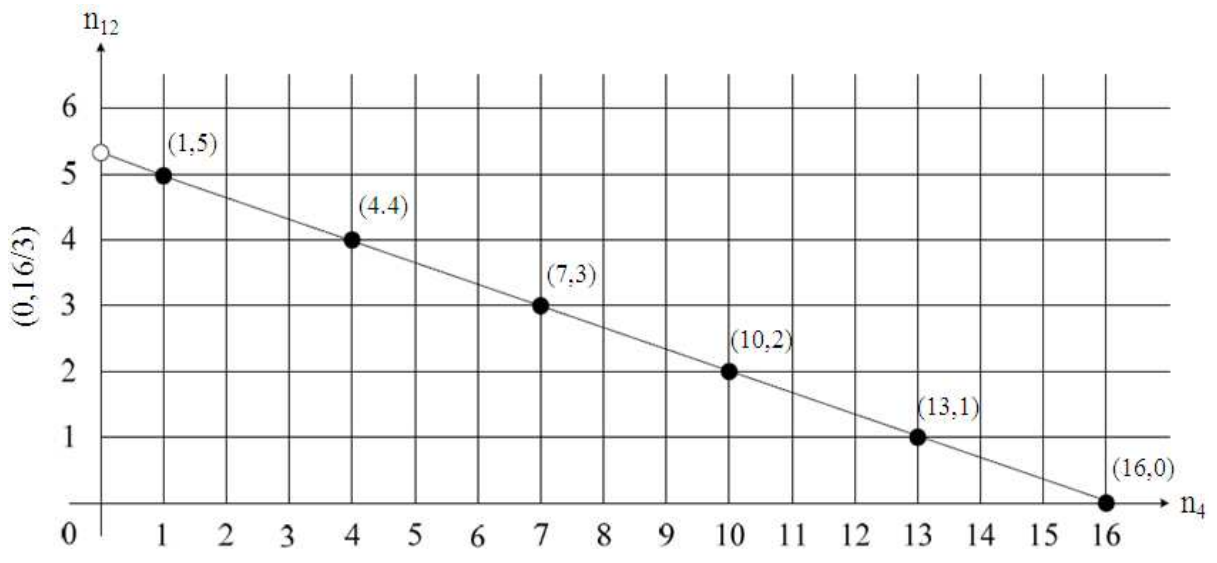

Fig. 6. Representation of the equation $n_{4}+3 n_{12}=16$ as a straight line in the two-dimensional space of $n_{12}$ versus $n_{4}$ Permissible nonnegative integer solutions of $\left(\mathrm{n}_{4}, \mathrm{n}_{12}\right)$ are highlighted

There are six possible solutions for the pair $\left(\mathrm{n}_{4}, \mathrm{n}_{12}\right)$, $(16,0)(13,1),(10,2),(7,3),(4,4)$ and $(1,5)$. The scalar equation technique has no explicit way for distinguishing between these six candidate solutions. The solution $\mathrm{n}_{4}=$ $1, \mathrm{n}_{12}=5$ cited by Heidel et al. (2003) has five distinct cycles of period twelve and one cycle of period four. This solution represents the actual network solution as can be verified by viewing the map of all possible trajectories for the network, as shown in Fig. 7.

Unfortunately, there is no immediate way to single out this desirable solution out of the mathematically valid six solutions. The scalar equation technique fails to identify the actual network solution without some implicit help or pre-knowledge from an exhaustive exponential-cost technique.

In the following, we employ some Boolean-equation techniques (Brown, 2003; Hammer and Rudeanu, 1968; Rudeanu, 1974; Rushdi, 2001; 2004) (as a supplement to the scalar equation method) to identify the states and the numbers of all period-four cycles. For this purpose, we augment the Equations (1) with the Equation (75a to f):

$$
\begin{aligned}
& \mathrm{A}(\mathrm{t}+4)=\mathrm{A}(\mathrm{t}) \\
& \mathrm{B}(\mathrm{t}+4)=\mathrm{B}(\mathrm{t}) \\
& \mathrm{C}(\mathrm{t}+4)=\mathrm{C}(\mathrm{t}) \\
& \mathrm{D}(\mathrm{t}+4)=\mathrm{D}(\mathrm{t}) \\
& \mathrm{E}(\mathrm{t}+4)=\mathrm{E}(\mathrm{t}) \\
& \mathrm{F}(\mathrm{t}+4)=\mathrm{F}(\mathrm{t})
\end{aligned}
$$

By virtue of Equation (67) and (75), we have Equation (76a to f):

$$
\begin{aligned}
\mathrm{A}(\mathrm{t}+4) & =1+\mathrm{F}(\mathrm{t}+3)=1+\mathrm{E}(\mathrm{t}+2) \\
& =1+\mathrm{D}(\mathrm{t}+1)=1+\mathrm{C}(\mathrm{t})=\mathrm{A}(\mathrm{t}) \\
\mathrm{B}(\mathrm{t}+4) & =\mathrm{A}(\mathrm{t}+3)=1+\mathrm{F}(\mathrm{t}+2) \\
& =1+\mathrm{E}(\mathrm{t}+1)=1+\mathrm{D}(\mathrm{t})=\mathrm{B}(\mathrm{t}) \\
\mathrm{C}(\mathrm{t}+4) & =\mathrm{B}(\mathrm{t}+3)=\mathrm{A}(\mathrm{t}+2)=1+\mathrm{F}(\mathrm{t}+1) \\
& =1+\mathrm{E}(\mathrm{t})=\mathrm{C}(\mathrm{t}) \\
\mathrm{D}(\mathrm{t}+4) & =\mathrm{C}(\mathrm{t}+3)=\mathrm{B}(\mathrm{t}+2)=\mathrm{A}(\mathrm{t}+1) \\
& =1+\mathrm{F}(\mathrm{t})=\mathrm{D}(\mathrm{t}) \\
\mathrm{E}(\mathrm{t}+4) & =\mathrm{D}(\mathrm{t}+3)=\mathrm{C}(\mathrm{t}+2)=\mathrm{B}(\mathrm{t}+1) \\
& =\mathrm{A}(\mathrm{t})=\mathrm{E}(\mathrm{t}) \\
\mathrm{F}(\mathrm{t}+4) & =\mathrm{E}(\mathrm{t}+3)=\mathrm{D}(\mathrm{t}+2)=\mathrm{C}(\mathrm{t}+1) \\
& =\mathrm{B}(\mathrm{t})=\mathrm{F}(\mathrm{t})
\end{aligned}
$$

The results in (76) can be combined to give Equation (77a and $b$ ):

$$
\begin{aligned}
& \mathrm{A}(\mathrm{t})=\overline{\mathrm{C}}(\mathrm{t})=\mathrm{E}(\mathrm{t})=\alpha \\
& \mathrm{B}(\mathrm{t})=\overline{\mathrm{D}}(\mathrm{t})=\mathrm{F}(\mathrm{t})=\beta
\end{aligned}
$$

Table 2 assigns all possible arbitrary values for the constants $\alpha$ and $\beta$ above and hence identifies all states on period-four cycles. The number of these states is four, which indicates the existence of a single period four cycle $\left(\mathrm{n}_{4}=1\right)$ and hence the existence of five period-twelve cycles $\left(\mathrm{n}_{12}=5\right)$. The four states in Table 2 can indeed fit into a cycle, as can be verified from Equation (67) or Fig. 7. 

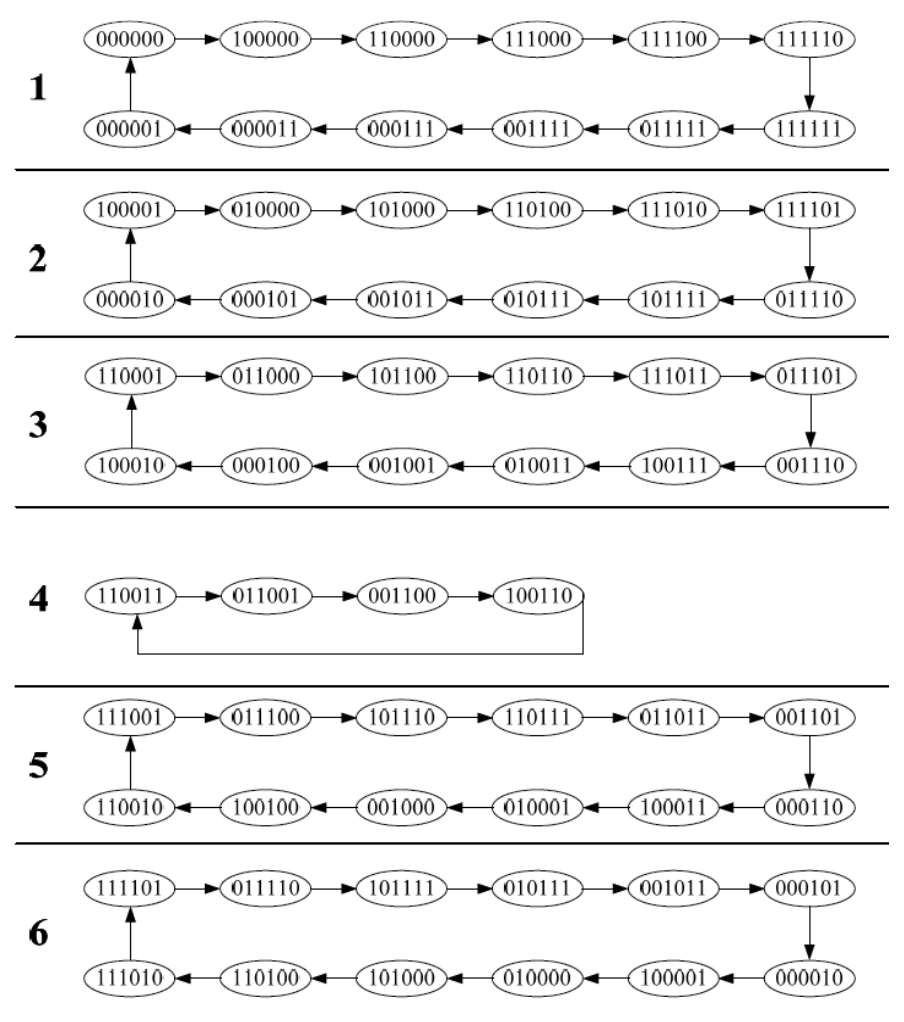

Fig. 7. A map of all possible trajectories for Example 3 showing five period-twelve cycles and a single period four cycle

Table 2. Solution for states on period-four cycles

\begin{tabular}{llllllll}
\hline$\alpha$ & $\beta$ & $\mathrm{A}(\mathrm{t})$ & $\mathrm{B}(\mathrm{t})$ & $\mathrm{C}(\mathrm{t})$ & $\mathrm{D}(\mathrm{t})$ & $\mathrm{E}(\mathrm{t})$ & $\mathrm{E}(\mathrm{t})$ \\
\hline 0 & 0 & 0 & 0 & 1 & 1 & 0 & 0 \\
0 & 1 & 0 & 1 & 1 & 0 & 0 & 1 \\
1 & 0 & 1 & 0 & 0 & 1 & 1 & 0 \\
1 & 1 & 1 & 1 & 0 & 0 & 1 & 1 \\
\hline
\end{tabular}

\section{DISCUSSION}

Derivation of the reduced scalar equation for a synchronous Boolean network is typically a cumbersome and time-consuming task. However, it could be somewhat simplified by adhering to the linear representation (Reed-Müller expansion) of the Boolean functions. Our examples show that this derivation is considerably facilitated by seeking and utilizing orthogonality relations among some successive instances of the same scalar Boolean variable. For some Boolean networks (see, e.g., Example 3), the reduced scalar equations are identical for all the Boolean variables. Our examples 1 and 2 demonstrate clearly that this is not always the case. In both examples, each variable has its own distinct reduced scalar equation.
There are infinitely many versions of a reduced scalar equation that are all equally suitable for deducing information about the network cycles or attractors. However, the true minimal reduced scalar equation is the only equation capable of predicting the transient behavior of the network. In some cases (see, e.g., Examples 1 and 2), this equation has a preceding version in which the pertinent times could be decremented by a single time period. Here, it is necessary to disprove this preceding version if one is to make sure that the reduced equation is truly minimal. In other cases (see, e.g., Example 3), the earlier time instant in the equation is the initial instant and cannot be decremented. Hence, no preceding version of the equation exists and there is no doubt about the minimality of the equation.

\section{CONCLUSION}

A description of a synchronous Boolean network is possible in terms of a linear reduced scalar equation which is the simplest two-term scalar equation that includes no Boolean operators and equates a latter value of a scalar variable $\mathrm{X}\left(\mathrm{t}_{2}\right)$ to an earlier value of the same variable $\mathrm{X}\left(\mathrm{t}_{1}\right)$, 
$t_{2}>t_{1}$. In this study, we achieved a better understanding of the nature of reduced scalar equations, demonstrated Boolean-algebraic techniques for deriving them, employed certain deduced orthogonality conditions for facilitating this derivation, presented other mathematical tools for utilizing the reduced scalar equations and finally reconciled the scalar methods with the more encompassing but more complex and less insightful matrix methods. In particular, we demonstrated, contrary to previously published assumptions or assertions, that there is typically no common reduced scalar equation for all the derivation of a reduced scalar equation necessitates validating it for the smallest instances $t_{1}$ and $t_{2}$ and hence is achieved not only by proving it but also by disproving an immediately preceding version of it when such a version happens to exist.

Since the disproof required for the equation preceding the minimal reduced equation is usually more tedious than the proof of the minimal equation itself, the reduced-scalar-equation method does not seem particularly helpful for evaluating transient length. Therefore, one might be content to quickly derive any reduced scalar equation, ignoring whether it is the true minimal version or a belated version and use this equation to study the cyclic behavior of the network. This strategy might make the most of reduced scalar equations, which are, as intended by Heidel et al. (2003), more transparent to analyze for cycles than the original matrix equations. There is a paramount interest in the study cycles in Boolean networks used as models of gene-regulatory network (Hopfensitz et al., 2013). As a network is trapped in a cycle as soon as one of its states is entered, cycles comprise the states in which the network resides most of the time. It is assumed that cycles in gene-regulatory networks are linked to phenotypes (Hopfensitz et al., 2013). Therefore, restricting the utility of scalar equations to cyclic behavior does not lead to a significant reduction of their importance.

\section{ACKNOWLEDGEMENT}

This article was funded by the Deanship of Scientific Research (DSR), King Abdulaziz University, Jeddah. The authors, therefore, acknowledge with thanks DSR technical and financial support.

\section{REFERENCES}

Andreescu, T. and D. Andrica, 2009. Diophantine Equations. In: Number Theory: Structures, Examples and Problems Andreescu, T. and D. Andrica (Eds.), Birkhäuser Boston, ISBN-10: 081763245X, pp: 145-165.
Andreescu, T., D. Andrica and I. Cucurezeanu, 2010. An Introduction to Diophantine Equations: A ProblemBased Approach. 1st Edn., Birkhäuser Boston, ISBN-10: 0817645489, pp: 345.

Brown, F.M., 2003. Boolean Reasoning: The Logic of Boolean Equations. 1st Edn., Courier Dover Publications, ISBN-10: 0486427854, pp: 291.

Cheng, D. and H. Qi, 2010a. State-space analysis of Boolean networks. IEEE Trans. Neural Netw., 21: 584-594. DOI: 10.1109/TNN.2009.2039802

Cheng, D. and H. Qi, 2010b. A linear representation of dynamics of Boolean networks. IEEE Trans. Automat. Control, 55: 2251-2258. DOI: 10.1109/TAC.2010.2043294

Cheng, D., 2009. Input-state approach to Boolean networks. IEEE Trans. Neural Netw., 20: 512-521. DOI: $10.1109 /$ TNN.2008.2011359

Cheng, D.Z., H. Qi and Z. Li, 2011. Analysis and Control of Boolean Networks: A Semi-Tensor Product Approach. 1st Edn., Springer, ISBN-10: 0857290975, pp: 488.

Cull, P., 1971. Linear analysis of switching nets. Kybernetik, 8: 31-39. DOI: 10.1007/BF00270831

Darby, M. and L. Mysak, 1993. A Boolean delay equation model of an inter-decadal Arctic climate cycle. Climate Dynam., 8: 241-246. DOI: 10.1007/BF00198618

Everest, G. and T. Ward, 2005. Diophantine Equations. In: An Introduction to Number Theory, Everest, G. and T. Ward (Eds.), Springer-Verlag, London, UK., ISBN-10: 1852339179, pp: 43-58.

Farrow, C., J. Heidel, H. Maloney and J. Rogers, 2004. Scalar equations for synchronous Boolean networks with biological applications. IEEE Trans. Neural Netw., 15: 348-354. DOI: 10.1109/TNN.2004.824262

Gregg, J., 1998. Ones and Zeros: Understanding Boolean Algebra, Digital Circuits and the Logic of Sets. 1st Edn., IEEE Press, ISBN-10: 0780334264, pp: 281.

Hammer, P.L. and S. Rudeanu, 1968. Boolean Methods in Operations Research and Related Areas. 1st Edn., Springer-Verlag, pp: 329.

Heidel, J., J. Maloney, J. Farrow and J. Rogers, 2003. Finding cycles in synchronous Boolean networks with applications to biochemical systems. Int. J. Bifurc. Chaos, 13: 535-552. DOI: 10.1142/S0218127403006765 
Hopfensitz, M., C. Mussel, M. Maucker and H.A. Kestler, 2013. Attractors in Boolean networks: A tutorial. Comput. Stat., 28: 19-36. DOI: 10.1007/s00180-012-0324-2

Huang, S. and I. Ingber, 2000. Shapedependent control of cell growth, differentiation and apoptosis: Switching between attractors in cell regulatory networks. Exp. Cell Res., 261: 91-103. DOI: 10.1006/excr.2000.5044

Milligan, D.K. and M.J.D. Wilson, 1993. The behavior of affine Boolean sequential networks. Connect. Sci., 5: 153-167. DOI: 10.1080/09540099308915693

Muroga, S., 1979. Logic Design and Switching Theory. 1st Edn., Wiley, ISBN-10: 0471044180, pp: 617.

Rudeanu, S., 1974. Boolean Functions and Equations. 1st Edn., North-Holland, ISBN-10: 0720420822, pp: 442.

Rushdi, A.M. and S.O. Al-Otaibi, 2007. On the linear analysis of synchronous switching networks. Eng. Sci. J., 18: 43-72.

Rushdi, A.M. and S.O. Al-Otaibi, 2008. On limitations of using scalar equations for analyzing synchronous Boolean networks. JKAU. Eng. Sci., 19: 41-49. DOI: 10.4197/Eng.19-2.3
Rushdi, A.M., 2001. Using variable-entered Karnaugh maps to solve Boolean equations. Int. J. Comput. Math., 78: 23-38. DOI: 10.1080/00207160108805094

Rushdi, A.M.A., 2004. Efficient solution of boolean equations using variable-entered karnaugh maps. JKAU. Eng. Sci., 15: 105-121.

Schroeder, M., 2009. Diophantine Equations. In: Number Theory in Science and Communication, Springer, Berlin, ISBN-10: 3540852972, pp: 119137.

Wilson, M.J.D. and D.K. Milligan, 1992. Cyclic behavior of autonomous synchronous Boolean networks: Some theorems and conjectures. Connect. Sci., 4: 143-154. DOI: 10.1080/09540099208946609

Zhao, Q., 2005. A remark on "Scalar equations for synchronous Boolean networks with biological applications. IEEE Trans. Neural Netw., 16: 171501716. DOI: $10.1109 /$ TNN.2005.857944 